\title{
Bun splitting: a practical cutting stock problem
}

\author{
Celia A. Glass • Jeroen M. van Oostrum
}

Published online: 13 November 2008

(C) The Author(s) 2008. This article is published with open access at Springerlink.com

\begin{abstract}
We describe a new hierarchical 2D-guillotine Cutting Stock Problem. In contrast to the classic cutting stock problem, waste is not an issue. The problem relates to the removal of a defective part and assembly of the remaining parts into homogeneous size blocks. The context is the packing stages of cake manufacturing. The company's primary objective is to minimise total processing time at the subsequent, packing stage. This objective reduces to one of minimising the number of parts produced when cutting the tray load of buns. We offer a closed form optimization approach to this class of problems for certain cases, without recourse to mathematical programming or heuristics. The methodology is demonstrated through a case study in which the number of parts is reduced by almost a fifth, and the manufacturer's subsidiary requirement to reduce isolated single bun parts and hence customer complaints is also satisfied.
\end{abstract}

Keywords Two-dimensional cutting stock problem · Multi-stage optimization · Bakery

\section{Introduction}

Bunny Bakery is a large manufacturer of bread, cakes and buns. Their bakeries produce hundreds of thousands of buns and rolls each day. Production is automated where possible, to such an extent that little room for efficiency improvements remains. However, the company was having problems with certain small bun and roll lines. The present authors were invited to investigate. The unexpectedly interesting mathematical problem that emerges from the investigation is the subject of this paper. The mathematical representation of the problem is unusual.

Despite Bunny Bakery's generally excellent reputation, an unacceptably large number of complaints had been received about damaged buns. The oven settings had already been

C.A. Glass $(\bowtie)$

Cass Business School, City University, 106 Bunhill Row, London EC1Y 8TZ, UK

e-mail: c.a.glass@city.ac.uk

J.M. van Oostrum

Econometric Institute, Erasmus University Rotterdam, P.O. box 1738, 3000 CA Rotterdam,

The Netherlands

e-mail: vanoostrum@few.eur.nl 
adjusted to minimize burning. We were therefore asked to look at the production stages after the buns emerge from the oven. The company's primary concern relates to total processing time of a bun production run. It will turn out that there are in fact other hidden variables which must also be optimized.

The context of production is as follows. After being baked in large tray-loads, the buns go through two further stages. Firstly, a block of attached buns is split into smaller parts. Any burnt or misformed buns are removed. Then, the smaller blocks of buns are assembled and packaged in standard size packs, ready for distribution to retailers. The two stages of splitting and packing are closely inter related, to the extent that a desirable outcome may be controlled at the bun splitting stage.

From a mathematical point of view, we are presented with a two dimensional Stock Cutting Problem. However, our problem, in which defective sections must be removed, is not of a standard form found in the literature. In our case, it is not the degree of waste that must be minimized, but rather (essentially) the number of parts. Moreover, two consecutive sets of 2D cuts are required. The first set is to reduce the tray load of buns into mainly homogeneous blocks or part blocks, of the size required for packaging. The objective for this stage of cuts relates to the position of the defective piece within a resultant block. The second set of cuts removes the defective piece from its block and produces non-homogenous parts. These parts are later assembled into packet size blocks. The cutting schemes applied to consecutive blocks are inter-dependent, being constrained to ensure that the resultant parts fit together into whole packages with no further cutting. Beyond this, the objective is to minimise the total number of parts produced by the two levels of $2 \mathrm{D}$-cuts. The problem extends the existing classification and requires a new solution method. In this paper, we formulate the problem more tightly within the context of the literature, and present a solution methodology for certain dimensions of the problem. We thus lay the foundation for a general methodology for arbitrary dimensions of the problem instance.

We describe our solution method using a case study of a particular bun package size and tray configuration. The company's current cutting strategy for the case study is used as a benchmark against which to measure performance. We present a careful analysis of the cutting options which take account of the position on the tray of a defective bun. We were able to recommend a particular cutting scheme, which depends on left-over bun parts which have not yet been packaged. This produces a remarkably good outcome, in which the number of bun parts is minimized. In addition, the more subtle problem of damaged buns is eliminated by balancing inventory of bun parts. The net result is a remarkably efficient outcome. Subsequent, we prove that similar analysis when applied to more general instances of package and tray sizes lead also to improvement compared to current cutting strategies.

The layout of the paper is as follows. In Sect. 2 we formulate our problem and put it in context by briefly reviewing the related literature on two dimensional stock-cutting problems and packing problems. In Sect. 3, we describe the logistics of production at Bunny Bakery, including the company's current working practices. We present a solution approach for the specific case study in Sect. 4 including analysis of the range of cutting options that leads to a recommended cutting strategy. In Sect. 5 we extend our approach by considering the general case, and solving the specific case of a single row packet output to optimality. Finally in Sect. 6 we offer some concluding remarks.

\section{Background}

The bun splitting and assembly problem, which is outlined in more detail in the next section, may be formulated as follows: 
Given positive integers $n, m, a$ and $b(n+m>2)$, for a sheet of dimension $a \cdot n$ by $b \cdot m$, and a set of positions $P=\{(x, y): 0 \leq x \leq b m-1,0 \leq y \leq a n-1\}$,

find a guillotine cutting scheme

for each position $(x, y) \in P$ of a single 1 by 1 defective piece on the sheet,

which isolates the defective piece $(x, y)$,

while producing rectangular parts that can be grouped into blocks of size $n$ by $m$, using the minimum total number of parts.

The nature of this problem is that of the Cutting Stock and Packing Problems. We now consider how our problem can best be classified in the context of the recently published typography of Wascher et al. (2007). After putting our problem in this context, we review the relevant literature in the area. Our problem proves to have several idiosyncratic aspects, which are identified below. Please note that we refer to the block size as number of rows by number of columns (as is conventional for matrices but not necessarily for graph coordinates).

The problem tackled in this article requires two, consecutive, sets of 2D-guillotine cutting schemes neither of which need be checker-board. The first cutting scheme is required to produce homogeneous, or near homogeneous, blocks from a single large object. The blocks are of whole, or exceptionally part, package size in the bun context. The selection of cutting schemes relates to the positioning of a given fault on the tray, within the resultant block. The fault is assumed to affect only a single 1 by 1 position. The second stage involves a 2D-guillotine cutting scheme for the single block. The cutting scheme must isolate the fault. In addition, it produces a heterogeneous set of (non-defective) parts which are required to be assembled into whole packages in the long-run. The objective is to keep the resultant number of parts to a minimum, subject to the above assembly constraint. The value put on a cutting scheme therefore depends upon the existing parts remaining in the system, and possibly upon certain assumptions about the future distribution of fault positions on the tray.

There are two main versions of the problem which we formulate. One involves the assumption of long runs with a random distribution of fault positions on the tray. The other more on-line approach is to take account of the combination of spare parts which will still remain after the cuts. A realistic alternative scenario, which is not considered in this article, is the restriction of faults to the edge of the tray.

The first stage, of this two stage problem, may be classified as a 2D rectangular Cutting Stock Problem, of a specific variety. In contrast to the literature its objective is that of minimising the number of parts resulting after both this and the next cutting stage, not that of minimising the waste. Alternatively, when one aims to position parts of package size on a tray, it can be viewed as a 2D rectangular Identical (or near identical) Item Packing Problem (IIPP), though the standard objective, of maximising the number of identical rectangular objects which are accommodated by a packing, is insufficient. Account needs to be taken of the positioning of the defective piece within an output object. Recall that the aim is to produce as few parts as possible while ensuring that they can all be fitted together into whole packages. From this perspective the trays are inter-dependent. The bun splitting problem can therefore be viewed as having several identical large items, which each have a defect, randomly positioned. The nearest category then becomes that of the Multiple Identical Large Object Placement Problem.

It appears, from Wascher et al. (2007) and the website of the special interest group of EURO on cutting and packing problems (ESICUP) that there is just one article available on 2-dimensional rectangular MILOPP, namely the paper of Hong et al. (1997). The MILOPP is an extension of the Single Large Object Placement Problem (SLOPP). This problem category is studied by several authors (Cui 2004; Lodi and Monaci 2003; Herz 
1972; Hifi 1997, 2001; Hifi and M'Hallah 2005; Yanasse and Morabito 2006; Yanasse and Katsurayama 2008). Although similar general model formulations were used, none of the previously mentioned authors have considered defects as well as multiple stages.

The second stage may also be classified as a 2D rectangular Cutting Stock problem with heterogeneous output parts. A cost is associated with part combinations. The cost is set to be prohibitively high if it precludes matching these parts with others into whole packages, at some point depending upon the context. All other combinations are costed simply by the number of parts. In contrast to the literature, the objective is that of minimising the number of parts resulting from a cutting scheme, not the waste. Ultimately, all non-defective parts (i.e. the undamaged buns) of the large object (i.e. the tray load of buns) will be used in packages, regardless of how they are cut out. In addition, the position as well as the shape of one of the output parts is predetermined. However, the combination of shapes of the other rectangular small parts is not. The existing subcategories of the Cutting Stock Problem in the literature, of input minimisation and output maximisation, relate to the case when the shape of the small objects is known in advance. The subcategories as currently defined do not therefore fit our context.

More classical approaches of the two-dimensional CSP can be found in papers of researchers as Gilmore and Gomory (1965, 1966), Christofides and Whitlock (1977), and Christofides and Hadjiconstantinou (1995) who have studied various solution approaches using for instance Dynamic Programming, Branch-and-Bound, and exact approaches. The continuous grow of the field have resulted in both survey papers and papers that introduce typologies of cutting and packing problems (Dyckhoff 1990; Dyckhoff et al. 1997; Sweeney and Paternoster 1992; Wascher et al. 2007).

Christofides and Hadjiconstantinou (1995) for instance consider the maximization the value of small rectangular pieces cut from a large rectangular sheet. They present a treesearch algorithm that is limited by the use of a bound based on a state space relaxation of a dynamic programming formulation of the problem. Hence, the problem is decomposed and solved in independent stages.

Gilmore and Gomory $(1965,1966)$ acknowledge in their classical papers already the existence of various variants of the two-dimensional CSP and its applications in industry. They argue that a linear programming formulation suffices since many practical problems add additional restriction to the problem such that the problem size substantially decreases. The bun-splitting problem is such a variant of regular 2-D CSP with guillotine cuts in several aspects. By far the most common objective in the literature is minimizing waste. We by contrast consider minimizing number of parts produced by a cutting scheme in addition to minimizing the number of small parts, equivalent to waste. In addition we consider the stage after the cutting stage as well. This stage is sometime called the packing stage or the assembly stage. Smith et al. (2000) discuss an example of how to cut glass in a batch production subject to capacity restrictions in a subsequent stage. He proposes a non-linear integer formulation in which the objective is to minimize waste. Second, the bun splitting problem is complicated by the occurrence of defects. Various researchers have studied cutting problems where defects occur starting with a theoretical analysis by Hahn (1968).

Later papers are application-oriented for example related to the forestry industry (Ronnqvist and Astrand 1998), paper industry (Aboudi and Barcia 1998), and textile industry (Sarker 1988). Some researchers handle more complicated concepts of defects in the form of multiple grades in sheets where positioning of lower grade sections within the end-product plays a role (Ronnqvist 1995; Sweeney and Haessler 1990). In this context the objective relates to either the amount of waste or the number of cuts. We consider the problem of removing a defective bun part, while minimizing the total number of parts, for which none 
of the above approaches is directly applicable. The amount and location of damage during baking is not known until the tray of buns emerges from the oven.

\section{Production at bunny bakery}

\subsection{Assembly line}

Manufacturing at Bunny Bakery is made up of long runs of typically 5 to 10 hours at the bakery. A single oven typically produces around 20,000 buns an hour. The output of an individual run consists of packages of a single type of bun of a particular size, e.g., 2 by 3 or 1 by 8 buns. The manufactory process we consider is represented in Fig. 1, and consists of three stages: the baking stage, the splitting stage, and the packing stage.

From the oven a conveyor belt moves towards the operator in the splitting stage. At this conveyor, trays from the oven with buns are placed. During the time on this conveyor belt a single operator will split the trays in the splitting stage. The way the splitting is done will be considered later. After being split, the pieces of buns continue on the conveyor up to the turntable of the packing stage. At the turntable three or more operators collect the buns to pack and combine them into the required package size. After being placed on a piece of card, the buns are placed on another conveyor toward a packing machine, which will handle them further. That part and the part further downstream will not be taken into consideration. The three different parts of the assembly line, which are of special interest, are described separately below.

\subsection{Baking stage}

The trays are semi-manually filled before the oven. During the filling of the trays the operator there can decide to add an extra row to the tray. This can result in a small variation of 'tray sizes', by which we mean the configuration of the buns on the bakery tray. Normally the configuration of the buns remains unchanged during a run. The possibility of changing the tray size is useful for the different products of the bakery. A block of buns comes out of the oven with sufficient indentation between buns to allow easy splitting of rows and columns.

Due to misplacing of buns on the tray or to uneven cooking in the oven, a small but significant number of trays contain one or more burnt of misformed buns. Not all trays are affected, although a substantial part actually did have faults. The majority of those contain just a single faulty bun. The position of defects on a tray depends upon the cause of the problem, and may be commonly around the edge, or, for other production runs, randomly positioned.

Fig. 1 A representation of the assembly line at Bunny Bakery

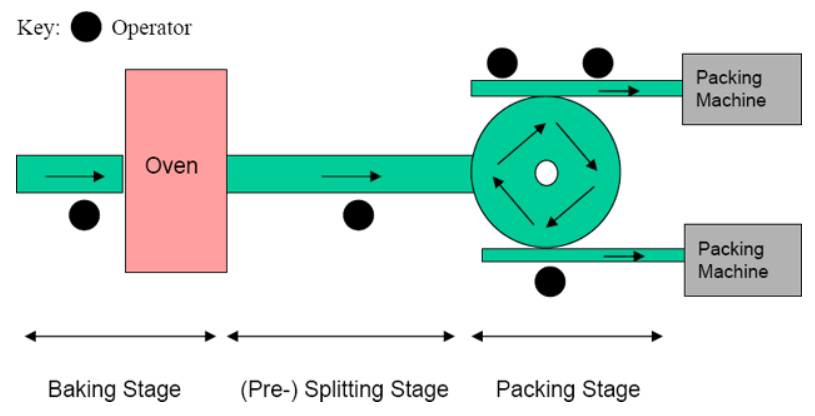




\section{3 (Pre-)splitting stage}

The trays with the buns from the oven are placed on a conveyor belt. They travel slowly through the splitting stage. There is generally one operator at this stage whose job it is to split the tray into smaller bun parts. The way this is done is in theory restricted by the time a tray of buns is in front of the operator, but the slow movement of the conveyor belt currently posses no such restriction. The trays come out of the oven one at a time and the operator handles each separately. The operator splits a tray of buns in one direction from the front before making any further splits.

\subsection{Packing stage}

In general there are three operators working at the packing stage. Their job is to assemble the buns on a piece of card ready for film wrapping and labeling by the packing machine. An operator puts a piece of cardboard in a predefined slot on the conveyor belt, before removing one or more pieces of buns from the turntable to cover the card. The configuration of buns in a packet varies with the product.

The turntable at the packing stage has a limited storage capacity of approximately ten trays worth of buns. The operators in the packing section cannot reach the whole table, as they can only reach the part in front of them. They have a strictly limited time to assemble the buns, and therefore pick the bigger parts first, which has the additional advantage of keeping the turntable as empty as possible. During a run the workload may vary due to breakdowns upstream and problems of any sort at the packing stage itself. Moreover, throughput of the ovens is slightly higher than that of the packers and it is thus unnecessary to aim for an empty turntable.

At the end of a run the operation changes a bit, the turntable must be emptied by the time a new run starts. To empty, as the turntable all the remaining parts, have to be combined into whole packages or else disposed of.

\subsection{Damaged buns}

The damage to a bun of which customers complain, occurs not during baking but at the packing stage. Burnt and misformed buns are already thrown away at the splitting stage. Subsequent damage is caused to the smaller parts that remain on the table for some time. The problem is most acute for the smallest, single buns, parts which are particularly susceptible to knocks from other parts on the turn-table. The potential for damage increases the longer a bun part remains on the turntable. In order to reduce the total cost of damaged buns, one would normally have to evaluate the nature of this cost increase with time far more precisely. However, the need for such an evaluation was alleviated by the results of our approach. As shown below we were able to eliminate single bun pieces all together.

The speed of packing depends on the number of blocks of buns, which need to be picked up and assembled into a single packet. The fewer the number, the higher is the speed. It seems to take only marginally longer to assemble a 2-block packet than a single block if both parts are easily to hand. However, assembling 3 or more blocks takes considerably longer. Thus, when production of blocks of buns is quicker than packing, the larger blocks will be removed first. What is observed of the current practice is the build up of single buns pieces. These can remain on the turntable for a long time and thus become damaged. It is these damaged parts which cause customer complaints, and hence result in additional costs for the bakery. A few buns get so damaged that they become waste. 
Fig. 2 Basic Cutting Scheme 1

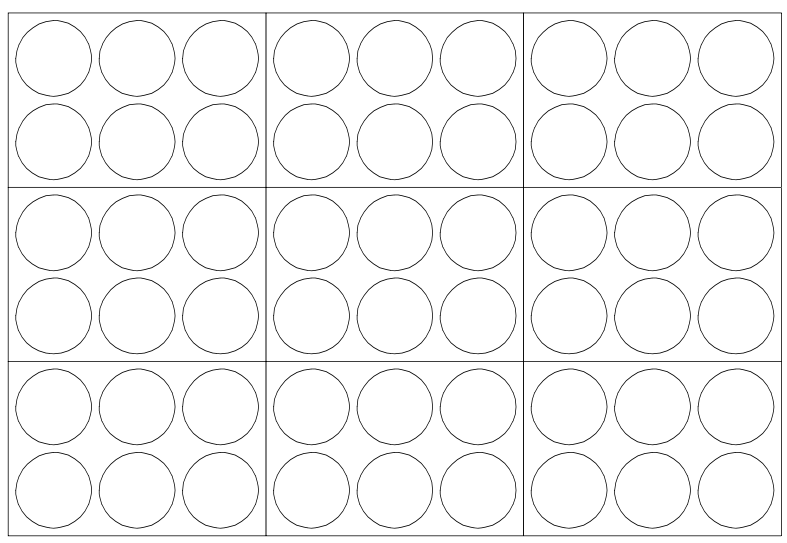

Fig. 3 Company's initial Sub-cutting schemes

\section{Corner fault}

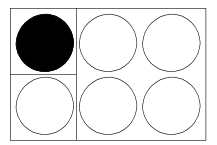

Sub-cutting scheme 1

\section{Non-corner fault}

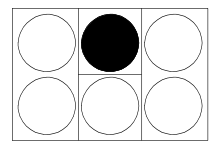

Sub-cutting scheme 2

\subsection{Working practice}

Each product line at Bunny Bakery has its own customized tray size and cutting scheme designed to facilitate package-size blocks of buns. Thus for example buns to be packed in 6 's are produced in tray loads of 9 rows and 6 columns and cut in a standard way into three $3 \times 6$ blocks each of which is further cut twice to achieve $3 \times 2$ blocks, as shown in Fig. 2 . We shall refer to this as Basic Cutting Scheme 1. The working practice at the bakery was to perform initial cuts at the splitting stage, in a regular pattern independent of defects In the past the splitting of the blocks to remove defect buns was done with the assembly of the smaller blocks at the packing stage, as indicated by the bakery's term of 'pre-splitting' for the preceding stage. The cutting pattern depends on the position of the bun in the block and takes one of two sub-cutting strategies, depending upon whether the faulty bun appears in a corner position of a $2 \times 3$ piece, as illustrated in Fig. 3 . However, the main bottleneck of the assembly process is the packing stage. In recognition, the work of cutting out defective buns has been moved to the earlier stage, which we refer to the throughout this paper as the 'splitting stage'.

\section{Developing cutting schemes for Bunny Bakery}

We now focus on how the production issues at Bunny Bakery, described above, can be translated into a mathematical model. The primary concern presented to us by Bunny Bakery was throughput, though the number of damaged buns is also a consideration. Our investigation of the production process, described above, reveals that the production bottleneck is the packing stage. This has consequences not only for throughput, but also for the quality of 
the product. The situation cannot be alleviated by further redeployment of staff, since the splitting stage is already operating with the minimum number of staff, namely one. We have reduced the problem to one of recommending cutting patterns for the splitting stage of production. The throughput issue is captured in the number of parts which need to be assembled at the packing stage. However, concern over damaged buns is an issue and translates into a secondary criterion relating to the length of time a bun block remains on the turntable at the packing stage, unpacked. The matching up of blocks into packages is therefore a consideration of any solution method. From a mathematical perspective, we are thus required to recommend a $2 \mathrm{D}$ guillotine cutting scheme which minimizes the number of parts it produces.

We approach the problem by undertaking a detailed investigation of the precise circumstances leading to bun damaged. One might need, for example, to examine the relationship between damage and length of time a bun spends, or the amount of congestion, on the turntable. However, such investigation proved to be unnecessary as we were able to reduce both of these factors as much as it is possible to do so. Our methodology is described for particular case at Bunny Bakery in this section. We return to the problem with more general dimensions in Sect. 5, where we give a more rigorous formulation and more detailed analysis.

\subsection{Approach}

Our methodology is best illustrated by way of a particular problem instance and for this purpose we use one of the scenarios from Bunny Bakery. The particular production line under consideration uses trays with 9 columns and 6 rows of buns, to produce buns for packing in a 3 by 2 configuration. We consider the case when there is one faulty bun on a tray. As mentioned in our problem formulation, the relevant part of the production process for us is the splitting (or cutting) stage, which needs to be coordinated with the subsequent packing stage.

The output produced after defective buns have been cut out, consisting of bun blocks of different sizes. For convenience we enumerate these in Table 1 . The bun blocks or parts need to be assembled into whole $3 \times 2$ packages during the packing stage. In order to assemble them into the maximum number of whole packages, we need to be aware of all possible combinations. These are therefore presented in Table 2.

The output of this splitting stage determines the speed of the bottleneck, packing stage. Damage to the final product relates to bun parts which remain on the turntable for a length of time. The two measures of interest are therefore, the total number of, and the combination of, parts produced from the tray-load of buns, after removal of a single defective bun. In particular, it is preferable to minimise the number of single bun parts produced.

Our approach is therefore to examine the combination of bun parts which are desirable for producing whole packets of buns. We then formalise the two stage cutting process required

Table 1 Shapes of parts for assembly

\begin{tabular}{ll}
\hline Part type & Size of the shape \\
\hline A & 3 by 2 \\
B & 2 by 2 \\
C & 3 by 1 \\
D & 2 by 1 \\
E & 1 by 1 \\
\hline
\end{tabular}


Table 2 The combinations of part types which can make up whole package of buns

\begin{tabular}{ll}
\hline Combination of part types & Number of part types \\
\hline A & 1 \\
B + D & 2 \\
$2 \times$ C & 2 \\
$3 \times D$ & 3 \\
$B+2 \times$ E & 3 \\
$C+D+E$ & 3 \\
$C+3 \times E$ & 4 \\
$2 \times D+2 \times E$ & 4 \\
$D+4 \times E$ & 5 \\
$6 \times E$ & 6 \\
\hline
\end{tabular}

for producing whole and part packets and removing defective buns. We start by considering the cutting strategy employed by Bunny Bakery themselves at the point we where called in, in Sect. 3.6. The company's initial cutting scheme will be used as a benchmark against which our recommendation is later evaluated. An extended range of cutting schemes is offered, in Sect. 4.2. This section thus lays the groundwork for a detailed analysis of the combined effect of different basic and sub-cutting schemes on the two relevant measures, leading to an optimal solution described in Sect. 4.4. We evaluate the outcome of this cutting strategy with the current working practice in Sect. 4.5. Our analysis throws light upon the build up of single buns observed in practice. It also serves to illustrate how our approach overcomes the problem. Section 4.6 concludes the case analyse of Bunny Bakery's cutting strategy.

\subsection{Full range of basic and sub-cutting schemes}

While the current cutting strategy described above is simple, it is observed to lead to a build up of single bun parts. Indeed analysis in Sect. 5.1 verifies this observation. A wider range of cutting strategies is therefore called for, and a fuller range of alternatives is now considered.

Basic cutting schemes separate mainly parts of package size from a tray. Besides Basic Cutting Scheme 1, four other schemes exist that produce 9 parts of package size. These are shown in Fig. 4. Further flexibility is offered by cutting scheme 6 and 7, shown in Fig. 5, which each produce 10 parts.

The purpose of making more than the first two one-dimensional cuts, described above, is to remove faulty buns. This is done by so-called sub-cutting. The various spare parts from different trays with faulty buns that appear after cutting are an important characteristic of a cutting scheme. Current practice provides two cutting strategies, sub-cutting schemes 1 and 2. The first one is carried out when the defect appears in the corner of a packet-size block and provides two smaller parts. The latter is used for non-corner defects and results in three left-over parts. It is not possible to produce fewer spare parts in either case. However, it is useful to have a broader choice of parts available for combining into whole packets at the packing stage. For this reason we propose two additional sub-cutting schemes, namely those numbered 3 and 4 shown in Fig. 6. Observe that the new schemes also produce two parts when removing a corner defect and three for a non-corner one. Thus, all else being equal, it is preferable to undertake a basic cutting scheme which allows the faulty bun to take a corner position. 


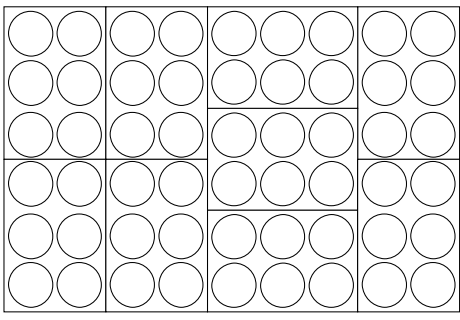

Basic Cutting Scheme 2

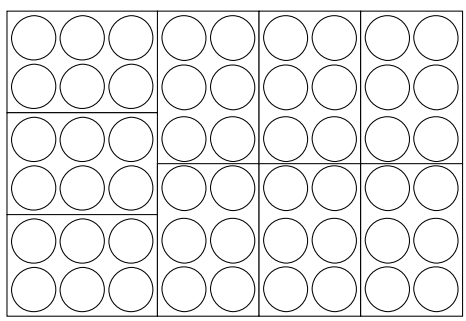

Basic Cutting Scheme 3

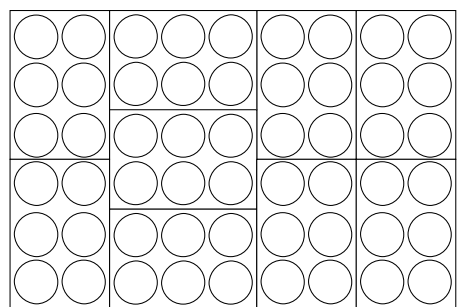

Basic Cutting Scheme 3

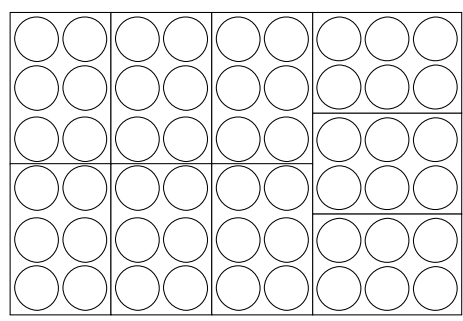

Basic Cutting Scheme 4

Fig. 4 Basic Cutting Schemes producing 9 parts

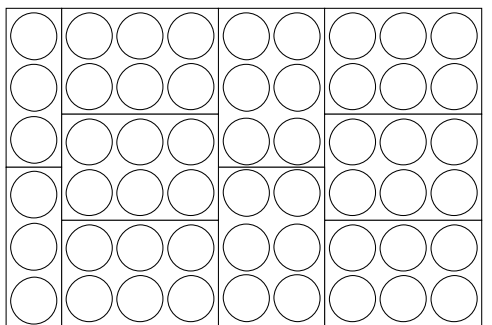

Basic Cutting Schemes 6

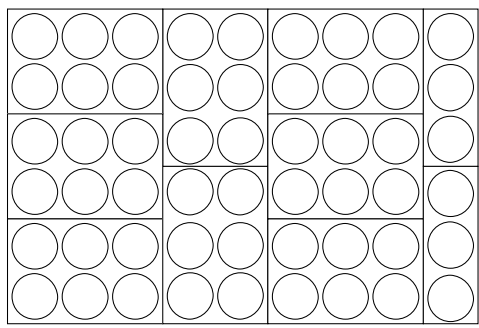

Basic Cutting Schemes 6

Fig. 5 Additional Basic Cutting Schemes producing 10 parts

Fig. 6 Additional possible Sub-cutting schemes

\section{Corner fault}

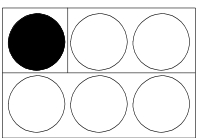

Sub-Cutting Scheme 3

\section{Non-corner fault}

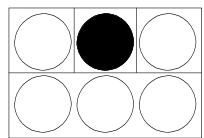

Sub-Cutting Scheme 4

\subsection{Default cutting strategy}

We first consider the effect of the cutting strategy which Bunny Bakery was using when they came to us. The working practice at the bakery was to perform initial cuts at the splitting stage, in a regular pattern independent of defects, as shown in Fig. 2. We shall refer to this 


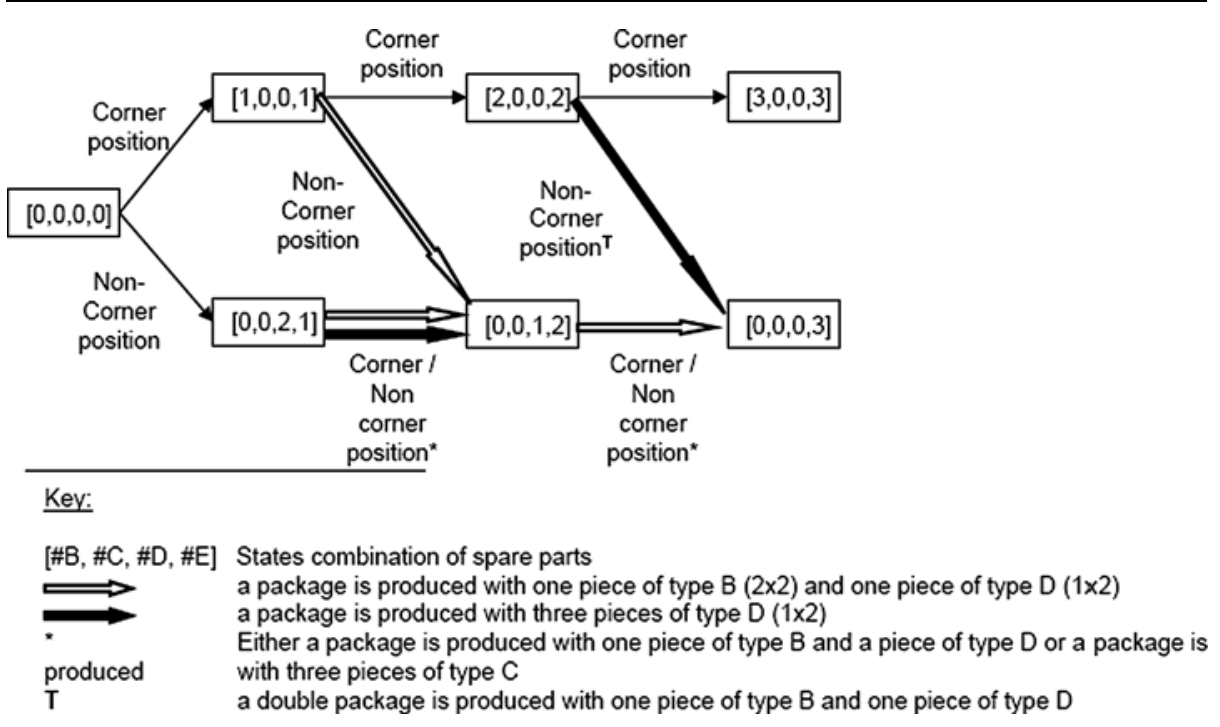

Fig. 7 Bun parts state diagram for Bunny Bakery's initial cutting strategy

as Basic Cutting Scheme 1. A defective bun is removed by carrying out guillotine cuts on the $3 \times 2$ block in which it occurs. The cutting pattern depends on the position of the bun in the block and takes one of two sub-cutting strategies, depending upon whether the faulty bun appears in a corner position of a $2 \times 3$ piece, as illustrated in Fig. 3 .

To show how a cutting strategy performs in practice we need to consider the cumulative effect of trays containing a burnt bun. The state of the system at any one time may be summarized by the number of bun parts of each of the four types that are smaller than a whole package. We thus have a four field state vector. Each time a tray containing a faulty bun presents itself, the state of the system potentially changes. These changes are illustrated in the state diagram in Fig. 7.

The company's cutting strategy does well with respect to producing a small number of bun parts, which matches up into packages. For a random distribution of faulty positions, the 4 buns and 2 buns parts would be in balance producing whole packages leaving the single buns to accumulate. The reason being that there are twice as many positions on the tray which appear as a corner after the basic cutting scheme, as do not. However, a single bun part is produced with each sub-cutting. Moreover, as splitting of bun blocks is not generally done before the end of a production run at the packing stage unless absolutely necessary, some $2 \times 2$ block may also accumulate. The combination of remaining parts after each subcut depends on the position of the faulty bun being in a corner or not on its package size block. This conforms observations at the bakery. To make improvements we will need to examine part combinations and alternative basic and sub-cutting schemes in some details.

\subsection{Optimal cutting strategy}

We now consider how a broad choice of cutting schemes can improve matters, and the extent to which the build up of single bun pieces may be avoided. The effect of a cutting scheme on a tray-load of buns depends not only on the position of the faulty bun on the tray, but also on the spare bun parts awaiting assembly into whole packets. The relevant factor here is whether the faulty bun ends up in a corner or a non-corner position of its packet size block. 
Fig. 8 Basic cutting scheme (as numbered in Figs. 4 and 6), which ensures a corner position for a faulty bun in corresponding position on the tray

\begin{tabular}{|l|l|l|l|l|l|l|l|l|}
\hline 1 & 3 & 1 & 1 & 2 & 1 & 1 & 2 & 1 \\
\hline 1 & 6 & 1 & 1 & 3 & 1 & 1 & 7 & 1 \\
\hline 1 & 3 & 1 & 1 & 2 & 1 & 1 & 2 & 1 \\
\hline 1 & 3 & 1 & 1 & 2 & 1 & 1 & 2 & 1 \\
\hline 1 & 6 & 1 & 1 & 3 & 1 & 1 & 7 & 1 \\
\hline 1 & 3 & 1 & 1 & 2 & 1 & 1 & 2 & 1 \\
\hline
\end{tabular}

Table 3 Characteristics of cutting scheme combinations

\begin{tabular}{|c|c|c|c|c|c|c|c|c|c|c|}
\hline \multicolumn{2}{|c|}{ Fault position } & \multicolumn{2}{|c|}{ Cutting scheme } & \multicolumn{6}{|c|}{ Output: Number of parts produced } & \multirow{2}{*}{$\begin{array}{l}\text { Number of whole } \\
\text { packages }\end{array}$} \\
\hline On tray & Within block & Basic & Sub & $\overline{\mathrm{A}}$ & B & $\mathrm{C}$ & $\mathrm{D}$ & $E$ & Total & \\
\hline Non-tricky & Corner & $1-5$ & 1 & 8 & 1 & 0 & 0 & 1 & 10 & 8 \\
\hline Non-tricky & Corner & $1-5$ & 3 & 8 & 0 & 1 & 1 & 0 & 10 & 8 \\
\hline Tricky & Non-Corner & $1-5$ & 2 & 8 & 0 & 0 & 2 & 1 & 11 & 8 \\
\hline Tricky & Non-Corner & $1-5$ & 4 & 8 & 0 & 1 & 0 & 2 & 11 & 8 \\
\hline Tricky & Corner & $6-7$ & 1 & 7 & 1 & 2 & 0 & 1 & 11 & 8 \\
\hline Tricky & Corner & $6-7$ & 3 & 7 & 0 & 3 & 1 & 0 & 11 & 8 \\
\hline
\end{tabular}

With basic cutting schemes 1 to 5, a faulty bun may be positioned in the corner of a bun block for all but four positions on the tray, as indicated in Fig. 8. The basic cutting schemes given in the diagram are not necessarily unique, but are sufficient for the purpose. We refer to the four positions which are not amenable to placing the faulty bun in a corner position of a package size sub-block using basic cutting scheme 1 to 5, as tricky positions.

The outcome of combinations of basic and sub-cutting schemes, in terms of type and number of parts, is enumerated in Table 3. For the non-tricky tray positions of a faulty bun, Basic Cutting Scheme 1 (or 2 to 5) combined with Sub-Cutting Scheme 3 dominates with respect to both objectives. That is, both total number of parts produced and the number of single buns produced are minimized. Similarly for the tricky tray position, it is possible to ensure that the faulty bun appears in a corner position of the resultant package-size sub-block without affecting the total number of parts. The possibility of a corner position allows us to choose a cutting scheme without resultant single bun parts. A recommended cutting strategy when faced with a fault in one of the four 'tricky' tray positions, therefore consists of Basic Cutting Scheme 6 (or 7) followed by Sub-Cutting Scheme 3. We will explore the effect of the strategy of adopting these schemes before considering any other. The only remaining consideration is the build up on spare parts.

A state diagram of the combination of spare parts resulting from this recommended cutting strategy is presented in Fig. 9. The diagram shows how the combination of spare parts rotates through six possibilities as trays with a faulty bun present themselves. Observe that the dynamics does not depend upon the fault position of consecutive faulty buns on the tray. The outcome does so well that there is no need to investigate further strategies. 


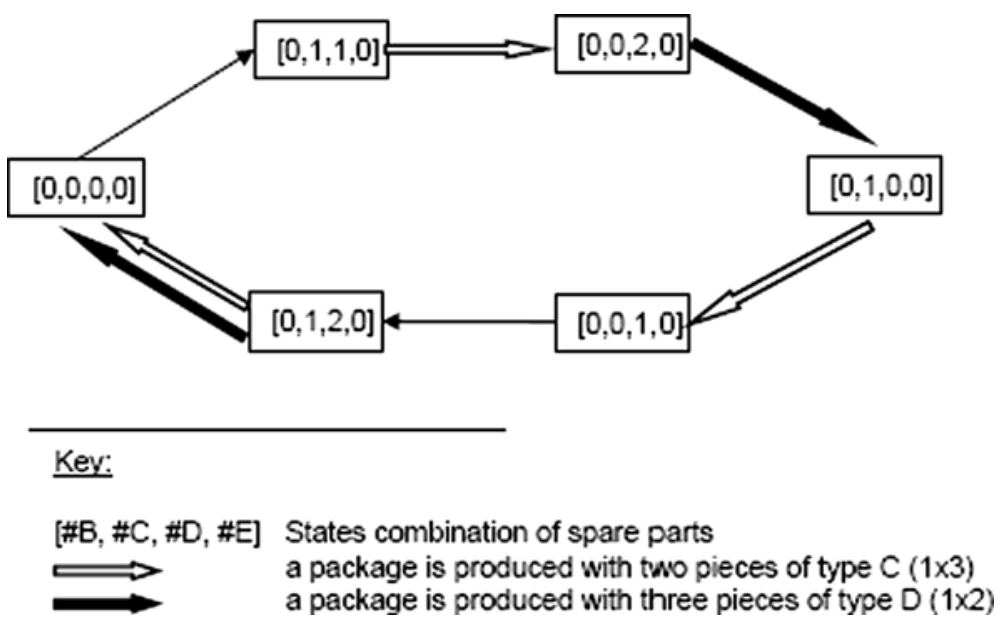

Fig. 9 Bun part state diagram for the recommended optimal cutting strategy

\subsection{Evaluation of results}

Under our optimal strategy, no single bun parts are produced. There is no need to trade off the total number of parts against the number of single bun parts produced. Both objectives are minimized simultaneously. However, we still need to explore the potential build up of spare parts.

The number of different bun part types produced is reduced from 4 to 2 in the optimal cutting strategy. The additional pair of $1 \times 3, \mathrm{C}$, parts produced when a defective bun is in a 'tricky' position on the tray, are paired up immediately. Aside from these, all trays containing a defective bun, produce one $1 \times 2$, D, part and a single $1 \times 3$, C, part. From the ten possible combinations, listed in Table 2, only two need arise in an optimal cutting strategy. At least half of the spare parts, namely the $\mathrm{C}$ parts, are matched up at the earliest opportunity, namely as soon as there is a defective bun on a tray. The rest are of type D which need remain on the turn table for no more than two such trays before combining into a complete packet. The turntable may thus be completely emptied of spare parts after every six trays with a defective bun, as shown in Fig. 9.

Bunny's previous cutting strategy comprises of a ratio of non-corner to corner position of 2:1. Given a random distribution of faults on trays, this cutting strategy leads on average to $4 / 3$ extra bun parts per single fault in comparison with a tray without faults. However, an optimal cutting strategy, presented in Sect. 4.4, leads to 58/54 extra bun parts per single fault, giving rise to the following proposition.

Proposition 1 For tray dimensions 6 by 9 and packet size 2 by 3, an optimal strategy reduces the number of extra bun parts produced due to a faulty bun is decreased by $19 \%$, compared to the default cutting scheme.

\subsection{Comments on methodology}

The simplicity of our approach relies upon the discrete nature of the objective function(s). Even with two objectives we are able to take advantage of being able to easily identify a dominant solution. The main work in identifying (pareto) optimal solutions for such a 
(bi-criteria) problem is therefore in the analyses of basic and sub-cutting schemes. This analysis may be carried out for any combination of tray and packet sizes. Our methodology is developed for a broader range of problem sizes in the next section.

In our case study, a single solution which is optimal with respect to the first criteria happened to also be so with respect to the second. Thus one can eliminate single buns while minimising the total number of bun parts. There is no guarantee of this being the case for other combinations of tray and packet sizes.

\section{The general problem and the case of single row output}

We now consider the broader class of problems, of which Bunny Bakery's bun splitting is an instance. These problems demands a two stage cutting scheme, each stage requires a set of 2D guillotine cuts, which need not conform to a checker board, i.e. the horizontal and vertical strips are independent. Since there is only a single defective piece, after the first two sets of guillotine cuts (one horizontal and for each strip one vertical) only one of the resultant rectangular blocks requires further cuts. The problem therefore naturally breaks down into two consecutive problems, each requiring a 2D-guillotine, non-check board, cuts.

Note that we consider using all parts to make up complete packs in the long run, and that the cutting scheme will depend upon the distribution of faults. In this article we assume hat faults are distributed uniform across the sheet. The integers $a$ and $b$ may be restricted to the form $m / \operatorname{gcd}(n, m)$ and $n / \operatorname{gcd}(n, m)$, respectively, as we discuss below.

Observe that in practice the tray size is usually a design decision and is made to be a multiple of $n \cdot m$, the block size. In this way, when a tray is free of defects, no package assembly is required after the guillotine cuts. Hence, the restriction on tray dimensions in the formulation above is applicable. Moreover, as there are further advantages to being easily able to cut rectangular blocks in either orientation from the tray, they tend to be designed with tray dimensions of the form $\frac{c \cdot n \cdot m}{\operatorname{gcd}(n, m)}$ by $b m$ or $a n$ by $\frac{d \cdot n \cdot m}{\operatorname{gcd}(n, m)}$, where $c$ and $d$ are integers. We may restrict analysis to tray size $\frac{n \cdot m}{\operatorname{gcd}(n, m)}$ by $n \cdot m$, for simplicity of analysis below.

\subsection{The hierarchical nature of the problem}

Now consider the nature of sub-cutting schemes. Cutting out a defective piece requires at least 2 guillotine cuts to a block (previously cut from the tray), and results in at least 2 smaller non-defective parts. Two smaller parts may be achieved only if the defective piece is at a corner of the block. If it lies along an edge, but not in a corner, at least 3 nondefective parts remain, while any central position results in at least 4 parts from any subcutting scheme. The essential reason for this relates to the number of sides of the defective piece which are attached to the rest of the block, and which therefore need to be cut following the first set of basic cuts.

The position of the defective part within a block depends upon the first stage of cuts, the Basic Cutting Scheme. Fewer smaller parts may be achieved by positioning a defect in a corner by a Basic Cutting Scheme, instead of elsewhere on the edge or more centrally. However, for some tray positions of the fault, the Basic Cutting Schemes, which do so, result in more of the larger parts. Moreover, given the relative position of a defective part on a block, whether the minimum number of parts can actually be achieved depends upon their shape relative to other left over parts. It is a strong requirement that the parts are assembled into whole blocks, even if this may require more cutting and results in additional parts. 


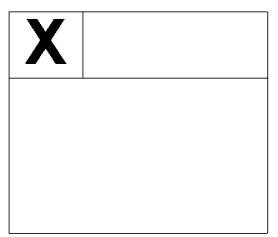

C1

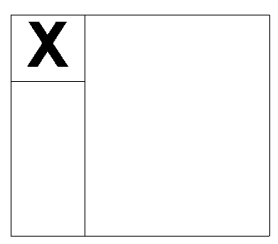

$\mathrm{C} 2$

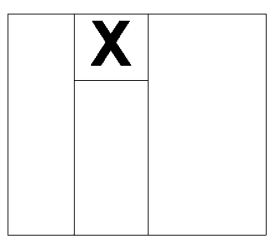

E2

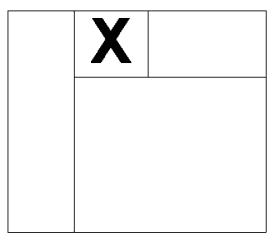

E3

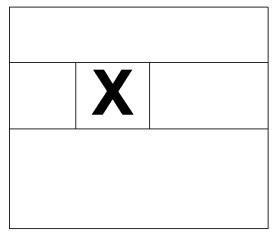

M1

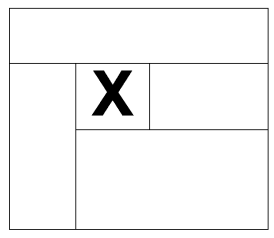

M2

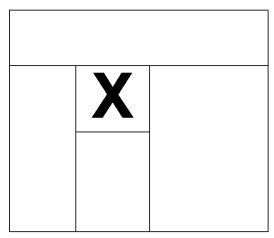

M3

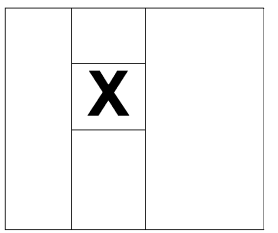

M4

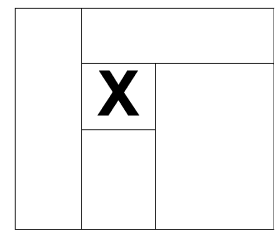

M5

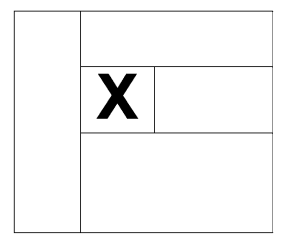

M6

Fig. 10 Sub-Cutting Schemes general case

\subsection{Default cutting strategy}

The regular Basic Cutting Scheme, with $b-1$ vertical guillotine cuts, followed by $a-1$ horizontal cuts to each of the $b$ parts of size $a \cdot n$ by $m$, may be considered the default and referred to as Basic Cutting Scheme (BCS) 1, as for the Bunny Bakery example in the previous section. We now consider the sub-cutting strategy which is appropriate in this case. A representative set of sub-cutting schemes is depicted in Fig. 10.

Proposition 2 Within the subspace of cutting schemes involving Basic Cutting Scheme 1, an optimal solution is achieved by adopting sub-cutting scheme C1, E1, or M1 as appropriate. The minimum average number of additional parts thus achieved is $\left(3-\frac{2}{n}-\frac{2}{m}\right)$ per defective tray with one defect, for uniformly distributed defects.

Proof We restrict our attention to the solution space in which the basic cutting scheme is the standard one, Basic Cutting Scheme 1. Consider the block with the defective piece. Suppose that we adopt sub-cutting schemes $\mathrm{C} 1, \mathrm{E} 1$ and M1 when the defect is at a corner, an edge or neither, respectively for trays with a single defective piece where the defects are equally likely to arise in any position on the tray. Now consider the $n$ by $m$ blocks, from multiple trays, with a defective piece in different positions. Assuming that the defects are randomly positioned, defects are uniformly distributed across the tray. Consider the $n$ defects which occur in each of the $m$ positions of row 1 (or row $n$ ), each tray gives rise to a single piece 
of size $(n-1)$ by $m$ and they together provide a set of single row strips of the following lengths: $m-1 ; 1$ and $m-2 ; 2$ and $m-3 ; \ldots, m-2$ and $1 ; m-1$. These strips pair up ( $m-1$ with $1, m-2$ with 2 etc.) to create $m-1$ strips of length $m$. Defects across row $i$, for $i \neq 1$ or $n$, give rise to $m$ pieces of size $(i-1)$ by $m$, and $m$ pieces of size $(n-i)$ by $m$, as well as a set of strips, as above, which when paired up provide $m-1$ strips of length $m$.

Now consider the combined result of parts resulting from a single occurrence of a defect in each position on an $n$ by $m$ block. The $m$ lots of pieces of size $(n-i)$ by $m$ and $(i-1)$ by $m$ for $i=1, \ldots, n$, can be matched up to make $m(n-1)$ packages of size $n$ by $m$. In addition there are $n$ by $(m-1)$ strips of length $m$ which provides $m-1$ additional packages, giving a total of $m \cdot n-1$ packages.

Thus, assuming uniform distribution of defects, over a long run, the set of parts from the above cutting strategy gives rise to $\frac{(\mathrm{nm}-1)}{\mathrm{nm}}$ whole packages, on average, with no further cuts. The number of cuts used to remove a part was in each case the minimum, as observed above. The sub-cutting scheme is therefore optimal among those whose basic set of cuts accords with Basic Cutting Scheme 1.

Defects arise in the positions on a package with uniform probability. Therefore a defective tray of size $a \cdot n$ by $b \cdot m$, from the above observation, at least produces

$$
a b-1+\frac{\{2 \times 4+3 \times 2(m+n-4)+4 \times(n-2)(m-2)\}}{n m}=a b+3-\frac{2}{n}-\frac{2}{m}
$$

parts by any choice of an appropriate sub-cutting scheme, on average. Whereas a nondefective tray will produce $a b$ parts.

\subsection{Optimization methodology}

Improvements upon the default cutting strategy described above can only be made when basic cutting schemes allow an otherwise edge positioned defective piece to be positioned at a corner of a block, or else an otherwise central piece to appear along an edge. In addition, resultant parts need to match up into whole packages or the gain in parts reduction may be lost. The approach is illustrated above for the Bunny Bakery $2 \times 3$ bun scenario with tray size $6 \times 9$. Our methodology is restricted by its nature to a case by case analysis, as it depends upon the relative dimensions of the problem. We illustrate the methodology further by considering further cases. The savings for each case, relative to that from the default cutting scheme described above, are summarized at the end of the section.

We start with packages consisting of a single row of buns, where $n=1$. We shall assume that the parameter $a$ is a multiple of $\frac{m}{\operatorname{gcd}(n, m)}$ for simplicity.

\subsection{Single row packages}

For single row blocks, when $n=1$, we take the tray size to be $m$ by $b \cdot m$, for simplicity. We first examine the default cutting scheme and show that it is adequate for minimizing the number of parts required to make whole packages, in the long run assuming that defects are uniformly distributed. However, this strategy results in many parts waiting for their complementary part to produce a whole package, even for uniformly distributed defects. Therefore, an alternative cutting scheme is recommended in order to avoid a build up of spare parts, when runs are short or defect positions distributed unevenly. The alternative cutting scheme outlined in Proposition 4 remains optimal regardless of fault position distribution for all trays of size $m$ by $b \cdot m$ with $b>1$. 
Proposition 3 When packages are a single row thick, of dimension 1 by $m$, a best subcutting scheme to follow Basic Cutting Scheme 1 is scheme C1 or E1 as appropriate, for uniformly distributed defects in the long run. It results in $(1-2 / m)$ additional parts per defective tray on average.

Proof The default cutting scheme described in Sect. 5.1 produces $m\left(3-\frac{2}{n}-\frac{2}{m}\right)=m-2$ additional parts for $m$ defective trays, by Proposition 2, when applied to the case of $n=1$.

Proposition 4 When packages are a single row thick, i.e., $n=1$, an optimal basic cutting strategy is to position the defective bun at the end of a strip. This may be achieved by a cutting scheme with $m$ horizontal strips of length $m$, one of which has the defective piece in a corner, and the remaining $(b-1) m$ strips vertical. This strategy is optimal, resulting in $1-1 / m$ additional parts per defective tray on average, regardless of the distribution of defects. In addition, at most one part remains in the system at any time.

Proof First observe that $m$ parts of length $m-1$ may be used to produce $m-1$ packages of size 1 by $m$ when one of the parts is divided into single pieces, using $m-2$ additional cuts. From Proposition 3 this is the minimum average number achievable. To achieve only pieces of length $m-1$ the defective piece must always be placed at the corner of a 1 by $m$ block by the basic cutting scheme. This is achieved by first cutting vertically at a distance of $m$ to one side of the defective piece, and singly elsewhere. The resultant $m$ by $m$ block is then cut $m-1$ times into $m$ horizontal strips. Finally the defective piece is removed by a single cut.

Observe that while the basic cutting scheme is dependent upon the position of the defect on the tray, the outcome, after sub-cutting, is not. This cutting strategy is therefore independent of the distribution in the position of the faults. Moreover, when there is a part remaining in the system from a previous batch, a piece of the appropriate length may be removed from the new part in the sub-cutting scheme to pair up with it to make a whole package. The system will thus be emptied after $m$ (defective) runs, and hold only one part during $m-1$ out of $m$ (defective) runs.

Now consider the secondary consideration related to the length of time which a part remains unpacked. The cutting strategy of Proposition 4 ensures that only one part will remain in the system. Moreover, each part remains in the system only the minimum length of time (i.e., until a subsequent tray contains a fault).

\section{Conclusions}

In this paper we have tackled a new type of $2 \mathrm{D}$ packing and cutting stock problem with defects. A solution involves two sets of 2D rectangular guillotine cuts which are not constrained to the checker-board variety. Not only are there two stages involved in getting to a solution, but the values of the solutions of a series of problems are interdependent, since the resultant parts from many sets needs to be reassembled into standard size blocks. The first set of cuts thus appears to be best classified, under Wascher et al. (2007) topography, as a Multiple Identical Large Object Placement Problem, MILOPP. There is little in the literature which addresses this type of problem. Moreover, it is useful to deviate from absolutely identical objects in our problem, and the value of a solution to the first stage of the problem depends upon that of the second stage. A defective part needs to be cut out during the second stage cutting process, restricting the set of possible cuts. In addition the problem differs 
from that in the literature in the criterion for the second stage cutting process which is not the classic one of minimising waste. It is the total number of parts resulting from both stages of the cutting process which is to be minimise over the series of runs.

We provide analysis for the case of a single row output of any length ( 1 by $m$ output parts) and for an instance of double row output, namely that of packages of length 3 (i.e., 2 by 3 output parts) with provably optimal solutions. The latter instance is solved in the context of the case study which led to this work.

The problem arises at the post-baking stages of a bun production line at Bunny Bakery. We showed how in this context problems which appeared to emanate from the packing stage could in fact be alleviated at the previous, splitting stage. The paper proposes a new type of solution method for finding optimum cutting strategies for this problem. Information on the inventory of the packing stage is used at the cutting stage to provide a good and easy fit of parts into packets and to avoid the production of any single part type. The bi-criteria problem of minimising the number of parts produced by the cutting scheme and the build up of spare parts awaiting assembly was effectively tackled for selected instances of the problem. For each circumstance, in terms of spare bun parts ready for assembly and the position of a defective bun on the tray, a particular cutting scheme is prescribed.

Our approach requires careful analysis. However, the recommendations can be carried out by the operator without recourse to a specialist optimization package or even to a computer. Hence, implementation is straightforward and requires only some time for instructing operators. The overall reduction in processing time, due simply to choice of cutting scheme, is estimated by Bunny Bakery at 5\%. The reduction in the number of extra parts produced by a faulty bun is $19 \%$. There are no hidden additional costs. The saving arises directly from the reduction in work at the bottleneck, packing, stage of production. A secondary, highly desirable, outcome is the dramatic reduction in the number of damaged buns since we were able to avoid the production of single bun parts. The problem of customer complaints concerning damaged buns could thus be more or less eliminated by improvements in the cutting design employed at the splitting stage. At the time of submission of this article, the approach is with Bunny Bakery for consideration.

The methodology we propose, while illustrated for a limited number of packets and tray size combinations, illustrating a general approach. We have thus introduced a new problem into the literature and offered an approach for small instances. It is beyond the scope of this paper to provide a comprehensive solution for the general case. Indeed it can be extended for pairs of faults and different bun configurations (van Oostrum 2004). We leave it for future research to take other combinations of dimensions with single faults and to develop a more general methodology. It can additionally be adapted to take account of an uneven distribution of fault position, e.g. restricted to the edge. The approach also applies in the context of similarly manufactured items other than bread buns and rolls.

Acknowledgements The authors thank Dr. G.F. Post (University of Twente) for many fruitful discussions. "Bunny Bakery" is a pseudonym for a company which is remaining anonymous for commercial reasons. We also thank colleagues at the company for suggesting the problem, and for continuing discussions during the course of this work. In addition, we thank two anonymous referees whose suggestions have contributed to the quality of this paper.

Open Access This article is distributed under the terms of the Creative Commons Attribution Noncommercial License which permits any noncommercial use, distribution, and reproduction in any medium, provided the original author(s) and source are credited. 


\section{References}

Aboudi, R., \& Barcia, P. (1998). Determining cutting stock patterns when defects are present. Annals of Operation Research, 82, 343-354.

Christofides, N., \& Hadjiconstantinou, E. (1995). An exact algorithm for orthogonal 2-D cutting problems using guillotine cuts. European Journal of Operational Research, 83, 21-38.

Christofides, N., \& Whitlock, C. (1977). An algorithm for two-dimensional cutting problems. Operations Research, 25, 30-44.

Cui, Y. (2004). Generating optimal multiple-segment cutting patterns for rectangular blanks. Proceedings of the Institution of Mechanical Engineers. Part B. Journal of Engineering Manufacturing, 218(11), 1483-1490.

Dyckhoff, H. (1990). A typology of cutting and packing problems. European Journal of Operational Research, 44, 145-159.

Dyckhoff, H. G., Scheithauer, J., \& Terno, M. (1997). Cutting and packing. In F. Dell'Amico, F. Maffioli, \& S. Martello (Eds.), Annotated bibliographies in combinatorial optimization (pp. 393-412). New York: Wiley.

Gilmore, P., \& Gomory, R. (1965). Multistage cutting stock problems of two and more dimensions. Operations Research, 13, 94-120.

Gilmore, P., \& Gomory, R. (1966). The theory and computation of knapsack functions. Operations Research, 14, 1045-1074.

Hahn, S. (1968). On the optimal cutting of defective sheets. Operations Research, 16, 1100-1114.

Herz, J. C. (1972). Recursive computational procedure for two-dimensional stock cutting. IBM Journal of Research and Development, 16(5), 462-469.

Hifi, M. (1997). An improvement of Viswanathan and Bagchi's exact algorithm for constrained twodimensional cutting stock. Computers \& Operations Research, 24(8), 727-736.

Hifi, M. (2001). Exact algorithms for large-scale unconstrained two and three staged cutting problems. Computer Optimization and Application, 16, 63-88.

Hifi, M., \& M'Hallah, R. (2005). An exact algorithm for constrained two-dimensional two-staged cutting problems. Operations Research, 53(1), 140-150.

Hong, L., Guangzhou, Z., \& Zongkai, L. (1997). A system of optimizing nesting with analogical learning mechanism. Computers \& Industrial Engineering, 32(4), 713-725.

Lodi, A., \& Monaci, M. (2003). Integer linear programming models for 2-staged two-dimensional Knapsack problems. Mathematical Programming Series B, 94, 257-278.

Ronnqvist, M. (1995). A method for the cutting stock problem with different qualities. European Journal of Operational Research, 83, 57-68.

Ronnqvist, M., \& Astrand, E. (1998). Integrated defect detection and optimization for cross cutting of wooden boards. European Journal of Operational Research, 108, 490-508.

Sarker, B. R. (1988). An optimum solution for one-dimensional slitting problems-a dynamic-programming approach. Journal of the Operational Research Society, 39, 749-755.

Smith, N. R., Al Khayyal, F., \& Griffin, P. (2000). A tree-search method for solving a cutting stock assignment problem. International Journal of Production Research, 38, 1557-1578.

Sweeney, P. E., \& Haessler, R. W. (1990). One-dimensional cutting stock decisions for rolls with multiple quality grades. European Journal of Operational Research, 44, 224-231.

Sweeney, P. E., \& Paternoster, R. E. (1992). Cutting and packing problems-a categorized, applicationorientated research bibliography. Journal of the Operational Research Society, 43, 691-706.

van Oostrum, J. M. (2004) Bun splitting (Technical Report Thesis). University of Twente, Enschede, The Netherlands, p. 62.

Wascher, G. H., HauBner, H., \& Schumann, H. (2007). An improved typology of cutting and packing problems. European Journal of Operational Research, 183, 1109-1130.

Yanasse, H. H., \& Katsurayama, D. M. (2008). An enumeration scheme to generate constrained exact checkerboard patterns. Computers \& Operations Research, 35, 2114-2128.

Yanasse, H. H., \& Morabito, R. (2006). Linear models for 1-group 2-dimensional guillotine cutting problems. International Journal of Production Research, 44(17), 3471-3491. 